\title{
TERMINO-LATERAL NEURORRHAPHY: THE FUNCTIONAL AXONAL ANATOMY
}

\author{
JASON M. ROVAK, B.S., ${ }^{1}$ PAUL S. CEDERNA, M.D., ${ }^{1 *}$ \\ VALDAS MACIONIS, M.D., ${ }^{2}$ MELANIE S. URBANCHEK, Ph.D., ${ }^{1}$ \\ JACK H. VAN DER MEULEN, Ph.D., ${ }^{1}$ and \\ WILLIAM M. KUZON, JR., M.D., Ph.D. ${ }^{1}$
}

The goal of this study was to determine the functional axonal anatomy of a termino-lateral neurorrhaphy (TLN). We hypothesize that axons populating a TLN must relinquish functional connections with their original targets prior to establishing new connections via the TLN. Two-month-old F344 rats underwent a TLN between the left peroneal nerve and a nerve graft tunneled to the contralateral hindlimb. Three months postoperatively, an end-to-end neurorrhaphy was performed between the nerve graft and the right peroneal nerve. Four months after the second operation, contractile properties and electromyographic (EMG) signals were measured in the bilateral hindlimbs. Left peroneal nerve stimula- tion proximal to the TLN site resulted in bilateral extensor digitorum longus (EDL) and tibialis anterior (TA) muscle contractions, with significantly lower forces on the side reinnervated by TLN. Evoked EMGs demonstrated that the right and left hindlimb musculature were electrically discontinuous following TLN. These data support our hypothesis that axons can form functional connections via a TLN, but they must first relinquish functional connections with their original targets.

2000 Wiley-Liss, Inc. MICROSURGERY $\quad 20: 6-14 \quad 2000$
Termino-lateral neurorrhaphy (TLN) or end-to-side nerve coaptation is a surgical technique for peripheral nerve reconstruction when the proximal nerve stump is not available for conventional end-to-end repair. In the late 1800s, TLN was originally utilized for the treatment of facial paralysis and brachial plexus injuries. ${ }^{1,2}$ Due to limitations in microsurgical instrumentation and technique, peripheral nerve reconstructions were largely ineffective and were abandoned. ${ }^{3}$ In the early 1990s, interest in TLN as a method of peripheral nerve reconstruction was revived. Experimentally, Viterbo et al. ${ }^{4}$ demonstrated muscle reinnervation via a TLN in the rodent model. Thereafter, numerous investigators ${ }^{5-8}$ have demonstrated functional muscle recovery following TLN in animal models. Clinically, TLN has been successfully used for facial reanimation and the treatment of sural nerve harvest site dysesthesia. ${ }^{9-12}$

Despite this promising clinical utility, a more complete understanding of the functional axonal anatomy of TLN is required before it can be widely applied in clinical situations. If axons populating a TLN must relinquish their original functional connections prior to establishing new con-

${ }^{1}$ The Department of Surgery and The Institute of Gerontology, University of Michigan, Ann Arbor, MI

${ }^{2}$ The Department of Plastic and Reconstructive Surgery, Vilnius University, Vilnius, Lithuania

*Correspondence to: Paul S. Cederna, M.D., Plastic and Reconstructive Sur gery, 2130 Taubman Health Care Center, 1500 E. Medical Center Drive, Ann Arbor, Ml 48109-0340. E-mail: cederna@umich.edu nections, then a donor nerve deficit may occur. If, on the other hand, collateral axonal sprouting from intact donor axons occurs, then the donor nerve deficit should be minimal. Understanding the functional axonal anatomy of TLN will help to maximize the functional recovery of muscles reinnervated by TLN, while minimizing the donor nerve deficits. The goal of this study was to determine whether individual axons that form functional connections via TLN can do so while maintaining their original connections, or whether donor axons must relinquish connections with their original targets. Specifically, we tested the hypothesis: Axons establishing functional neuromuscular connections via a TLN must relinquish functional connections to their original targets.

\section{MATERIALS AND METHODS}

\section{Animal Model}

Experiments were performed using 2-month-old, male, specific pathogen-free (SPF) F344 rats with a mean body mass of $290 \pm 30 \mathrm{~g}$ (Charles River Laboratory, Kingston, NY). Long isogenetic tibial nerve grafts $(8-10 \mathrm{~cm})$ were harvested from a separate group of adult male SPF F344 rats. All animal care, housing, and operative procedures were conducted in accordance with the National Institute of Health Guide for the Care and Use of Laboratory Animals (NIH Publication Number 85-23, 1985); the experimental protocol was approved by the University Committee on the Use and Care of Animals. Rats were housed individually or 
in pairs in a pathogen-free facility in the Unit for Laboratory Medicine at the University of Michigan, given food and water ad libitum, and exposed to a 12-h light-dark cycle. For all surgical procedures, rats were anesthetized with an initial intraperitoneal injection of sodium pentobarbital $(60 \mathrm{mg} / \mathrm{kg})$ and supplemented as necessary to maintain a deep plane of anesthesia. All nerve graft procedures were performed by the same surgeon. All surgery was conducted under aseptic conditions.

\section{Experimental Design and Paradigm}

The peroneal nerve innervating the extensor digitorum longus (EDL) and tibialis anterior (TA) muscles was isolated through a lateral thigh incision. An epineurial window was then created in the left peroneal nerve $15 \mathrm{~mm}$ proximal to the EDL muscle as described by Viterbo et al. ${ }^{9}$ Individual fascicles were visualized through the window but were not deliberately disturbed. A TLN was performed under an operating microscope (Zeiss OpMi-6, Carl Zeiss, Inc., West Germany) by placing 10-0 nylon interrupted epineurial sutures between the external epineurium at the site of the epineurotomy in the left peroneal nerve and the external epineurium at one end of the isogenetic tibial nerve graft. During this coaptation, an attempt was made to avoid injury to the fascicles visible through the epineurial window. The free end of the long tibial nerve graft was then tunneled subcutaneously to the contralateral hindlimb. The wound was closed in layers with 4-0 chromic sutures.

After the initial surgical procedure, a 3-month recovery period allowed for axonal regeneration through the TLN and tibial nerve graft. After the first recovery interval, the right peroneal nerve was exposed through a lateral thigh incision and then sharply divided $15 \mathrm{~mm}$ proximal to the EDL muscle. The proximal stump was resected to the sciatic notch and buried in the gluteus musculature. The distal end of the isogenetic tibial nerve graft was then coapted to the end of the distal stump of the right peroneal nerve using a standard epineurial technique (Fig. 1). The wound was closed in layers. A second, 4-month recovery period allowed for axonal regeneration and muscle reinnervation prior to measuring bilateral hindlimb contractile properties.

A group of adult (8-10-month-old) male SPF F344 rats did not undergo any operative procedures and functioned as the control group; maximal tetanic isometric force $\left(\mathrm{F}_{0}\right)$ was measured for the EDL and TA muscles of both hindlimbs.

\section{Measurement of Contractile Properties}

In situ measurements of force production were made in the right and left EDL and TA muscles 4 months after the second procedure in a manner similar to that previously described. ${ }^{13-16}$ Animals were anesthetized and the right and left EDL and TA muscles were isolated without injuring the neurovascular pedicles. The distal tendons of the EDL were identified on the dorsal surface of the foot, divided, and

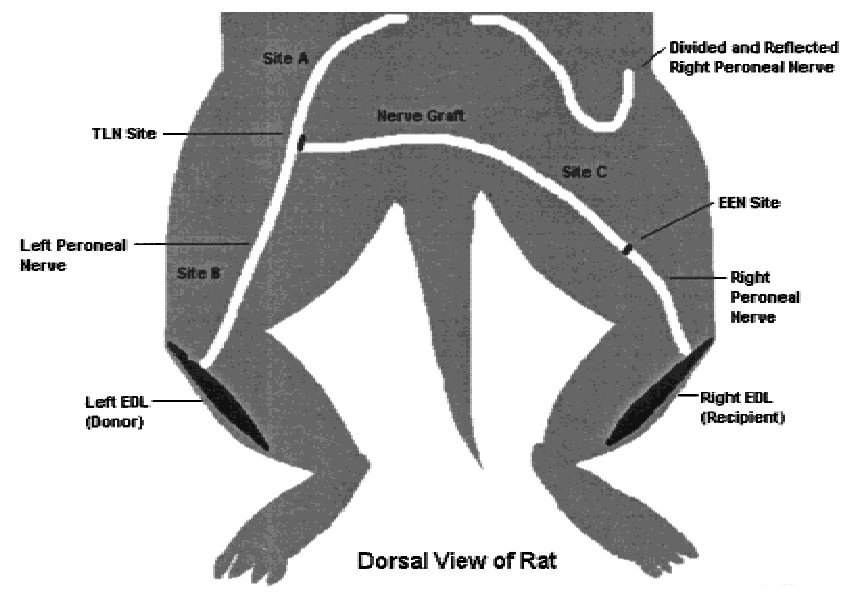

Figure 1. Dorsal view of the rat demonstrating the location of the $T L N$ and EEN sites. The nerve graft is interposed between the TLN and EEN sites.

folded to create a tendon loop. The tendon loop was secured at the musculotendinous junction with a 4-0 silk sutures; the loop was later used to secure the distal tendons to the force transducer (Kulite Semiconductor Products, Leonia, NJ). Due to the restrictive length of the TA tendon, a distal tendon loop could not be created. The distal tendon was identified in the anterior compartment of the hindlimb, divided, and secured with a 2-0 silk suture ligatures at the musculotendinous junction. The silk suture ligature was then tied directly to the force transducer. To avoid motion artifact from the contractions of adjacent muscle groups during the in situ measurements, the tibial and sural nerves were divided above the level of the knee. In addition, the distal tendons of the peroneus, gastrocnemius, soleus, and plantaris muscles were divided. The innervation of the EDL and TA muscles was preserved. The rats were then placed on a platform maintained at $37^{\circ} \mathrm{C}$ by a circulating water bath. The leg was immobilized during force measurements using an 18 gauge needle to secure the femoral condyle and a clamp to secure the foot to the platform. The EDL or TA was secured to the force transducer. Throughout the evaluation, the exposed muscles and nerves were regularly bathed with warm mineral oil; muscle temperature was monitored and maintained between $35^{\circ} \mathrm{C}$ and $37^{\circ} \mathrm{C}$.

The muscles were activated indirectly via supramaximal nerve stimuli (square pulses, $0.2 \mathrm{~ms}$ pulse duration, 4-6 V) generated by a Grass S88 Stimulator (Grass Instrument Co., Quincy, MA). The stimuli were delivered to the left peroneal nerve, proximal to the TLN coaptation site, using a shielded bipolar silver wire electrode (Harvard Apparatus, South Natick, MA). Force output at the musculotendinous junction was measured by the Kulite transducer, displayed on a storage oscilloscope (Gould Inc., Romulus, MI), and sampled via an analogue to digital converter (Data Translation, Marlboro, MA) interfaced with a microcomputer (Dell Computer Corp., Austin, TX). Custom software was 
utilized for data collection and signal processing (Asyst Software Technologies, Inc., Rochester, NY). Twitch contractions were used to determine the optimum muscle length for force production $\left(\mathrm{L}_{0}\right)$. With the muscle set at this length, a single $300-\mathrm{ms}$ stimulation of $50 \mathrm{~Hz}$ was delivered and $\mathrm{L}_{0}$ was again checked. All subsequent isometric force measurements were made at $\mathrm{L}_{0}$. Using digital calipers, $\mathrm{L}_{0}$ was measured directly as the total length of the muscle belly, excluding the tendons of origin or insertion. During singletwitch contractions, peak twitch force $\left(\mathrm{F}_{\mathrm{t}}\right)$ was measured. A force-frequency curve was then constructed by stimulating for $250 \mathrm{~ms}$ at increasing frequencies from 30 to $300 \mathrm{~Hz}$. Two minutes elapsed between tetanic contractions to permit muscle recovery. The maximal tetanic isometric force $\left(\mathrm{F}_{0}\right)$ was defined as the force plateau.

\section{Electromyography}

The anterior compartment muscles of both hindlimbs were activated indirectly with nerve stimuli (square pulses, $0.2 \mathrm{~ms}$ pulse duration, 3 second train duration, $3 \mathrm{~Hz}$ ) generated by the Biologic-Traveler Electrodiagnostic Testing System (Biologic Systems Corporation, Mundelein, IL) and sequentially delivered to each of three sites using a shielded bipolar silver wire electrode (Harvard Apparatus): (1) the left peroneal nerve proximal to the TLN site (Site A); (2) the left peroneal nerve distal to the TLN site (Site B); and (3) the tibial nerve graft distal to the TLN site (Site C)(Fig. 1). The minimum current necessary to elicit an electromyographic (EMG) response was utilized for all evaluations. As the various nerve locations were stimulated, EMG signals were recorded from the right and left hindlimb anterior compartment muscles using two stainless steel, unshielded, bipolar recording electrodes placed proximally and distally to the anterior compartment musculature. A stainless steel ground wire was positioned between the recording electrodes in adjacent soft tissue. EMG signals were displayed on the storage oscilloscope. The presence or absence of an EMG signal was determined in the anterior compartment musculature of the hindlimbs. This qualitative assessment was used to determine electrical continuity across the TLN and end-to-end neurorrhaphy (EEN) sites, but not to evaluate the efficiency of muscle reinnervation utilizing this technique.

After completion of the force and EMG measurements, the EDL and TA muscles were harvested, the tendons were trimmed, and the muscles were weighed. Muscles were then covered with cryopreservative and frozen with isopentane cooled by liquid nitrogen $\left(-160^{\circ} \mathrm{C}\right)$ and stored at $-60^{\circ} \mathrm{C}$ for subsequent histochemical processing. The right and left peroneal nerves and long tibial nerve grafts were harvested and preserved in a $3 \%$ glutaraldehyde, $0.1 \mathrm{M}$ sodium cacodylate solution for subsequent histomorphometric analysis. At the completion of each experiment, the animal was euthanized with an overdose of sodium pentobarbital.

\section{Histochemical Analysis}

Ten-micron thick cross sections of the EDL and TA muscles were cut in a cryostat (Microm, Waldorf, Germany). The muscles were stained with hematoxylin and eosin (H \& E) and myosin ATPase (mATPase) at five different $\mathrm{pH}$ levels $\left(10.4,9.4,4.5,4.3\right.$, and 3.8).${ }^{17-20}$ Muscle fibers were classified as either slow oxidative (SO), fast oxidative glycolytic (FOG), or fast glycolytic (FG), based upon differential staining to mATPase. ${ }^{21,22}$ Ten-micron thick cross sections of the epon-embedded tibial nerve graft were obtained using a microtome (DuPont, Wilmington, DE). The nerves were stained with Toludine Blue using standard techniques. ${ }^{23}$ A qualitative assessment of the muscle fiber type composition and spatial organization was performed on all muscles. The myelination and distribution of axons in the tibial nerve graft were similarly evaluated.

\section{Data Analysis}

The mean and standard deviation of maximum tetanic isometric forces were calculated for both the TA and EDL muscles from both legs. The effect of the surgical procedure on force production was determined by comparing right and left hindlimb EDL and TA muscle force production using Student's $t$-test. Statistical computations were performed using a microcomputer and Microsoft Excel (Microsoft Corp., Seattle, WA). The level of significance was set a priori at $P<0.05$.

\section{RESULTS}

Ten animals were entered into the study, completed both operations, and were available for contractile property measurements and EMG evaluations. Seven rats successfully completed the contractile property measurements and were available for EMG evaluations. Bilateral EMG evaluations were completed in four rats; three rats were excluded from the analysis due to technically inadequate EMG studies. All rats tolerated the initial surgical procedures well and demonstrated no long-term gait disturbances; no fixed joint contractures were observed. The mean masses of the EDL and TA muscles were significantly larger on the left compared with the right; no significant right-left difference in $\mathrm{L}_{0}$ was identified for either muscle.

Five control rats completed the contractile property measurements and were available for analysis. There were no significant differences in body mass or $\mathrm{L}_{0}$ identified between the control and TLN rats. Muscle mass was significantly larger in the control rats compared with the TLN $\operatorname{rats}(P<0.05)$.

\section{Force Measurements}

Isometric force measurements are summarized in Table 1. Left peroneal nerve stimulation proximal to the TLN site 
Table 1. Hindlimb $F_{o}$ Measurements in Control and Experimental Muscles ${ }^{\dagger}$

\begin{tabular}{lccc}
\hline Muscle & Control & \multicolumn{1}{c}{ Left (donor) } & Right (recipient \\
\hline EDL & $3,900 \pm 430$ & $3,300 \pm 400^{*}$ & $380 \pm 310^{*, * *}$ \\
TA & $11,900 \pm 1,000$ & $8,500 \pm 1,600^{*}$ & $500 \pm 440^{*, * *}$ \\
\hline
\end{tabular}

${ }^{\dagger}$ Data are displayed as mean \pm standard deviation of mean. *Statistically significant vs control muscle $F_{o}(P<0.05)$.

${ }^{*}$ Statistically significant vs. left (donor) muscle $F_{o}(P<0.001)$.

resulted in bilateral EDL and TA muscle contractions. The maximum isometric twitch $\left(\mathrm{F}_{\mathrm{t}}\right)$ and tetanic $\left(\mathrm{F}_{0}\right)$ forces were significantly lower in the right, compared to the left, EDL and TA muscles. A significant force deficit was identified in the EDL and TA muscles from the TLN rats compared with the control rats $(P<0.05)$.

\section{EMG}

EMG results are summarized in Table 2. Electrical discontinuity was identified between the right and left hindlimbs: (1) stimulation of the left peroneal nerve, proximal to the TLN site (site A), evoked EMG signals in the right and left EDL and TA muscles; (2) stimulation of the left peroneal nerve, distal to the TLN site (site B), evoked electrical signals in the left EDL and TA muscles only; and (3) stimulation of the long tibial nerve graft, distal to the TLN site (site C), elicited electrical activity in the right anterior compartment musculature only (Fig. 1).

\section{Histochemical Analysis}

A significant change in muscle fiber spatial organization and composition was identified in the right EDL and TA muscles reinnervated by TLN (Figs. 2 and 3). Specifically the right EDL and TA muscles exhibited pronounced fiber type grouping, a greater percentage of SO muscle fibers, and atrophic fibers, which are consistent with previous evaluations of reinnervated muscle..$^{24-31}$ No fiber type grouping or atrophic muscle fibers were identified in the left EDL or TA muscles.

Qualitative assessment of the long tibial nerve graft demonstrates numerous large myelinated axons populating the graft (Fig. 4).

\section{DISCUSSION}

The electrical discontinuity between the anterior compartments in the two limbs supports our hypothesis that axons establishing functional neuromuscular connections via a TLN must relinquish functional connections to their original targets. Note that our data cannot distinguish between two potential mechanisms by which this ultimate neuroanatomy may arise. It is possible that collateral sprouting of intact "donor" axons through the TLN occurs, but if the sprout forms a functional connection via the TLN, then the original target is relinquished by pruning of the contrac-
Table 2. EMG Results*

\begin{tabular}{lcc}
\hline & \multicolumn{3}{c}{ Recording site } \\
\cline { 2 - 3 } Stimulation site & Left EDL & Right EDL \\
\hline A & + & + \\
B & - & + \\
C & + & - \\
\hline
\end{tabular}

${ }^{*}+$ Indicates electrical continuity between the stimulating and recording electrodes; - Indicates no electrical continuity between the stimulating and recording electrodes.

tile synapse. It is also possible that donor axons were transected at the time of TLN, regenerated through the coaptation site, and subsequently reinnervated the contralateral hindlimb. The force deficits identified in the left (donor) EDL and TA muscles following TLN support the hypothesis that functional neuromuscular connections are sacrificed following TLN, but this information does not help to distinguish between the two potential mechanisms. However, the purpose of this experiment was not to define the mechanism by which axons populate a TLN, but rather to determine the functional continuity of axons in the "donor" and "recipient" nerves following TLN.

Several aspects of the experimental design strengthen our conclusions. First, we utilized a cross-leg nerve graft, reinnervating muscles in the hindlimb contralateral to the TLN. By designing a model in which the donor nerve and muscles reinnervated by TLN were in separate limbs, we reduced the possibility that autoreinnervation contributed to the functional outcome. The contractile and electrical activity that was observed in the right hindlimb following left peroneal stimulation could only be a function of axons populating the TLN graft. Several previous investigations have employed TLN grafts between a donor nerve and target muscles in the same leg. 4,7,32,33 These studies cannot exclude the possibility that autoreinnervation by the original nerve contributed to the functional results.

Second, our study investigated functional as opposed to anatomical connectivity following TLN. We confirmed, using muscle contractile property measurements, EMG, and muscle histochemistry that functional neuromuscular synaptic connections were formed by axons regenerating through the TLN graft. Although the functional recovery of the TA and EDL muscles after reinnervation of the right hindlimb was modest, the observed force generation in right hindlimb muscles from stimulation of the left, contralateral peroneal nerve is definitive evidence of functional axonal regeneration via the TLN graft. Recent double-labeling studies have demonstrated donor axon collateral sprouting following TLN. ${ }^{34}$ Although this anatomic continuity of individual axons in both the TLN graft and the donor nerve would be required if the axons were able to maintain connections to two disparate targets, anatomic continuity alone does not demonstrate that the collateral sprouts will successfully make functional connections with a new target 


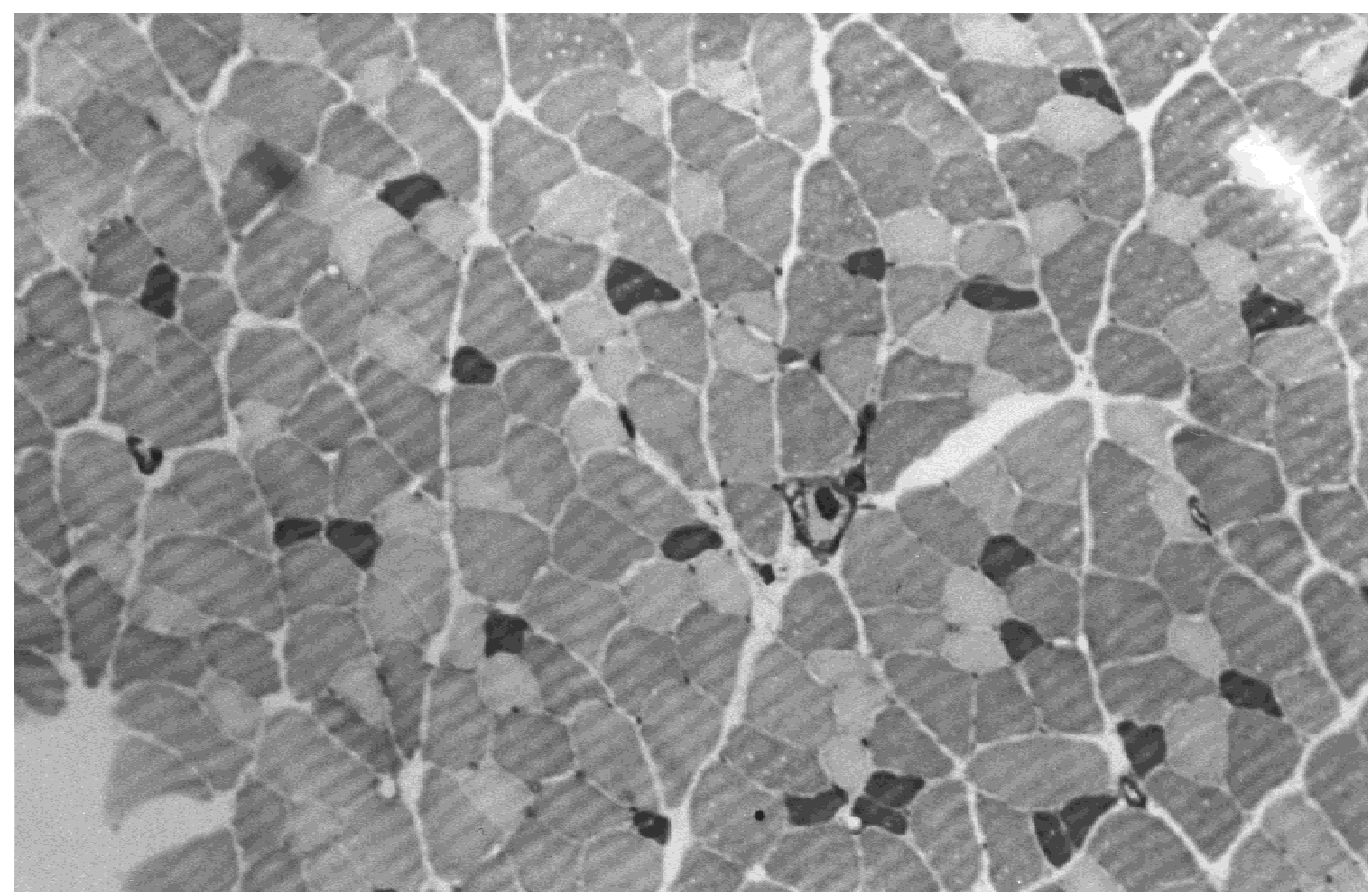

A

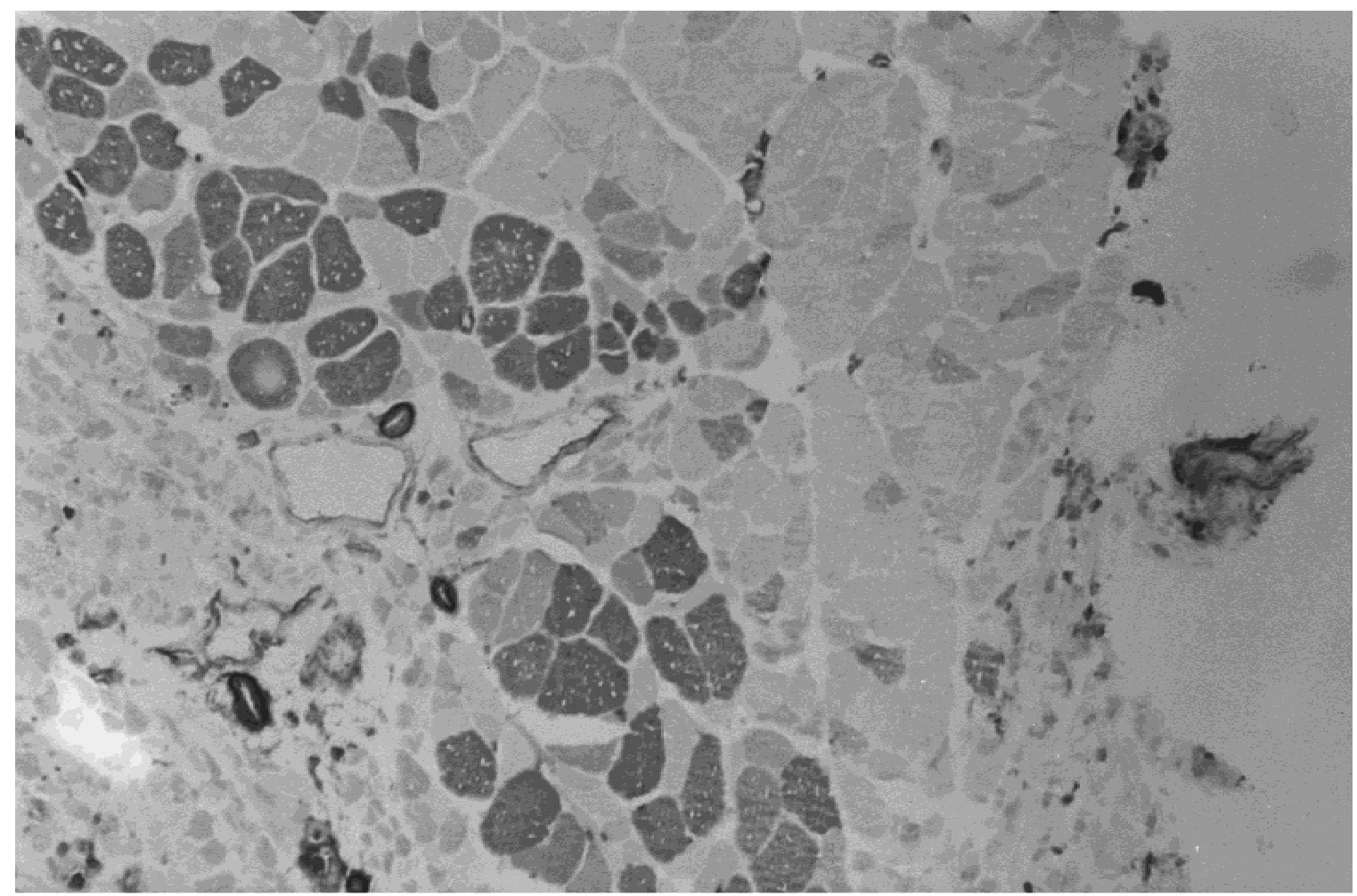

B

Figure 2. Photomicrograph (X100) of EDL muscles stained for mATPase (pH 4.5). Slow fibers stain darkly, fast fibers stain lightly. A: Left (donor) EDL muscle demonstrating normal fiber type composition and spatial organization. B: Right (recipient) muscle reinnervated by TLN demonstrating an increased percentage of slow fibers and fiber type grouping. 


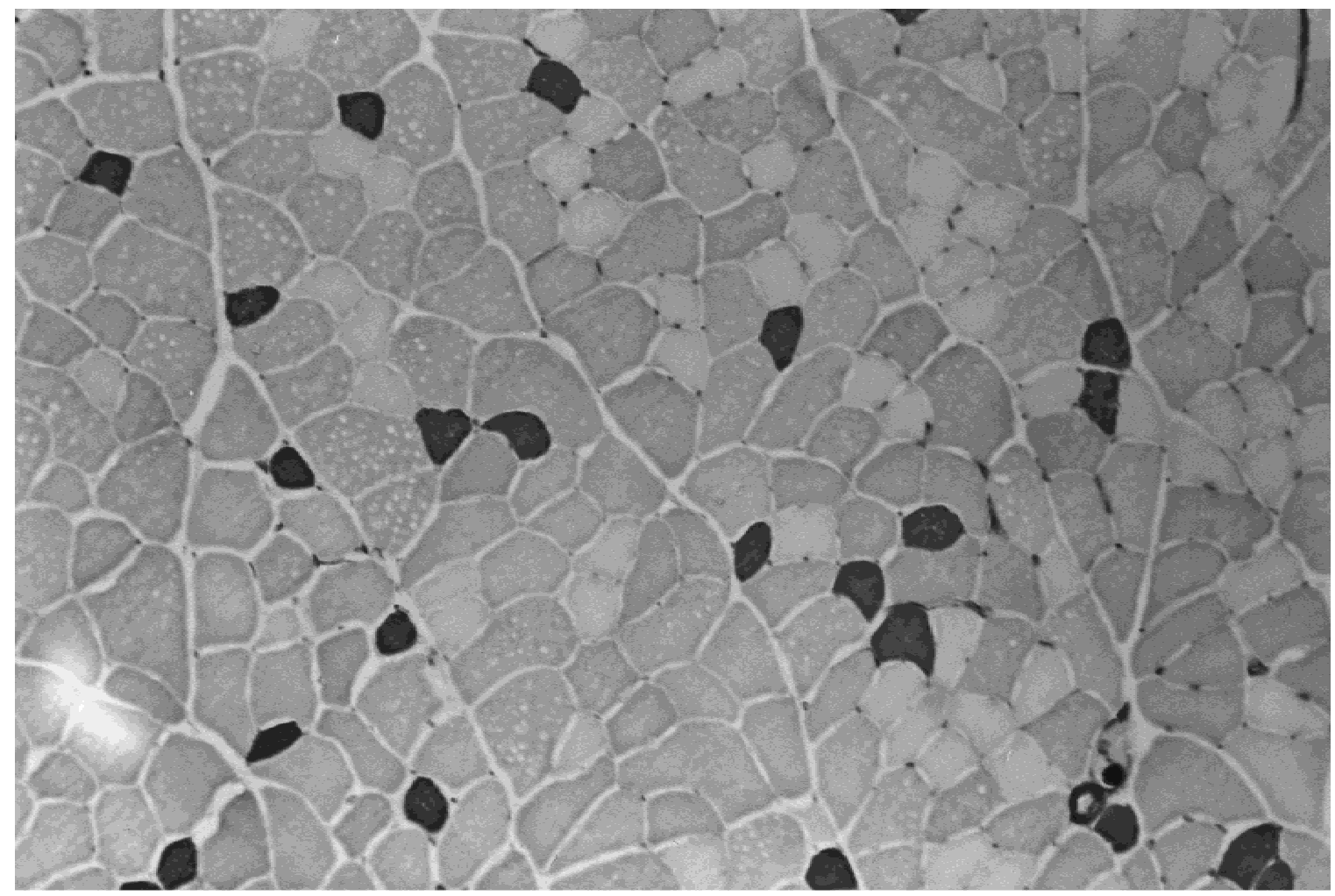

A

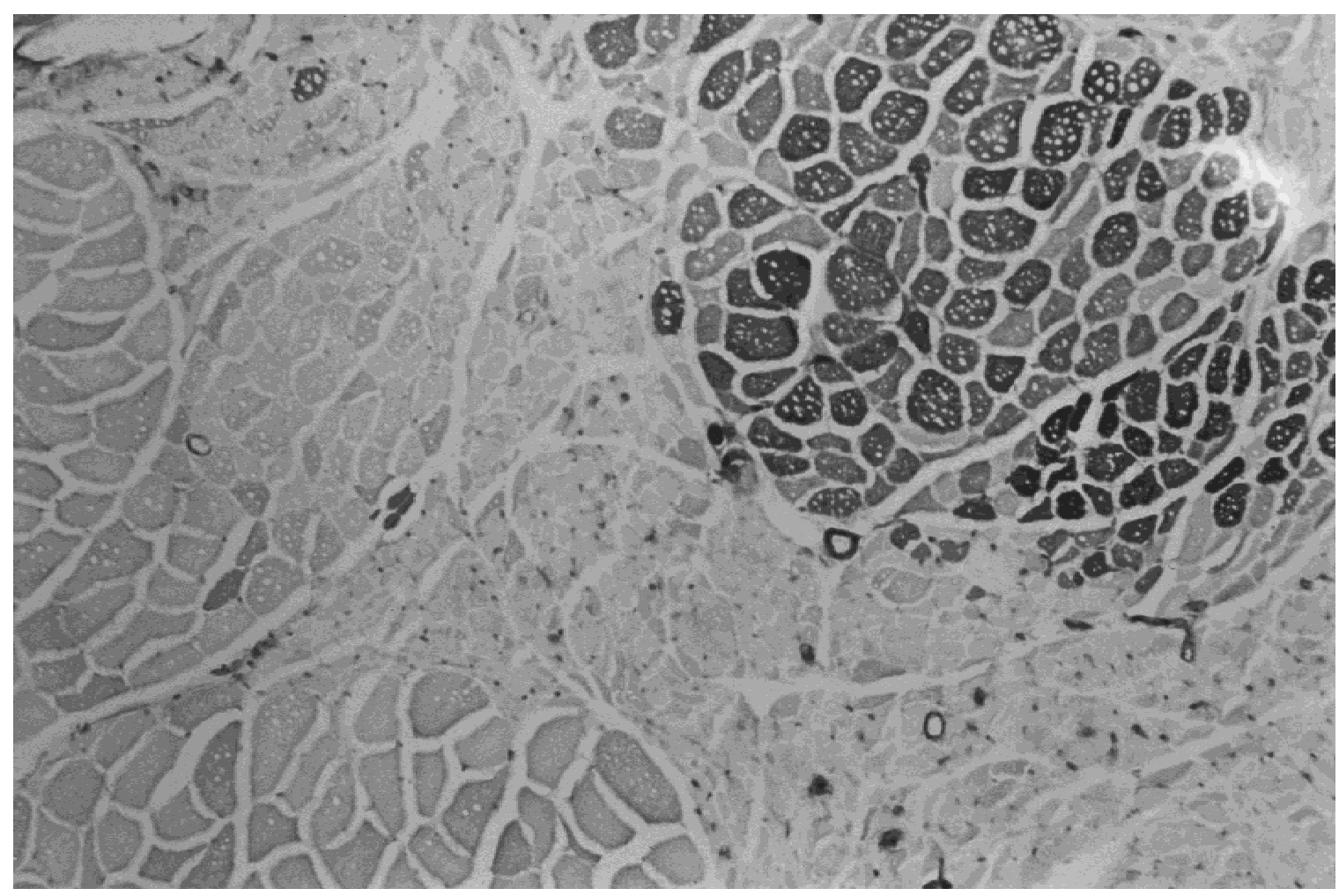

B

Figure 3. Photomicrograph (X100) of TA muscles stained for m-ATPase (pH 4.5). Slow fibers stain darkly, fast fibers stain lightly. A: Left (donor) TA muscle demonstrating normal fiber type composition and spatial organization. B: Right (recipient) TA muscle reinnervated by TLN demonstrating an increased percentage of slow fibers and fiber type grouping. 


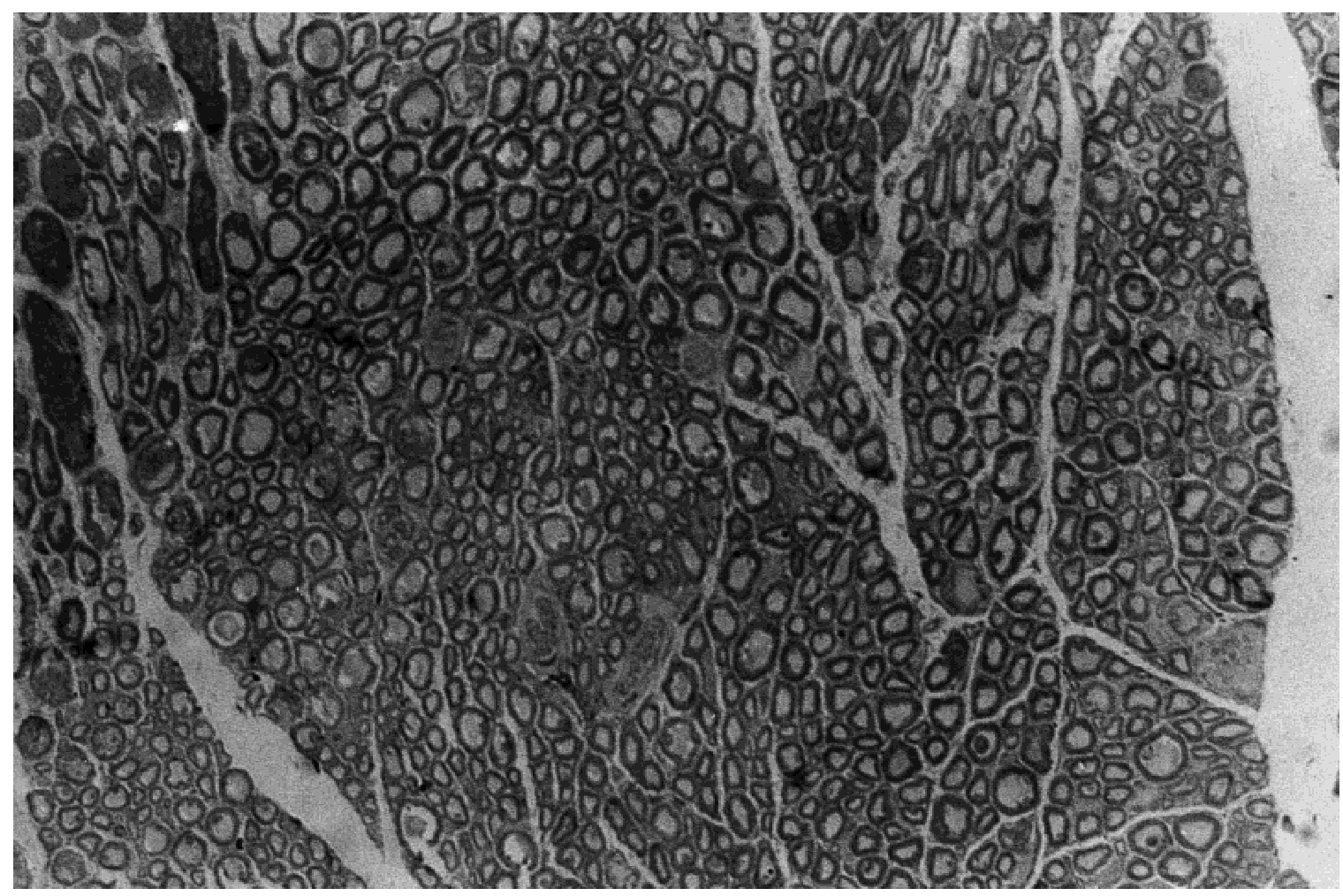

Figure 4. Photomicrograph (X250) of cross-leg tibial nerve allograft. Multiple myelinated axons are demonstrated.

muscle, or that a single axon can simultaneously maintain motor unit territories in more than one hindlimb muscle. The lack of electrical continuity between the two hindlimbs in our experiment supports the notion that if collateral sprouting occurs, either the parent or daughter branch is ultimately pruned. This conclusion is strengthened further by the chronicity of our experiments; the functional outcome of the TLN and cross-leg tibial nerve graft was evaluated 7 months postoperatively. In the double-labeling experiment referenced above, the experimental time course was much shorter, ranging from 2 to 8 weeks. ${ }^{34}$ For these reasons, our functional results are not inconsistent with previous anatomical studies.

Despite these factors which support our conclusions, several limitations in our study must be addressed. First, it is possible that branch point blocking could have affected our EMG results. Branch point blocking is a stimulation frequency-dependent phenomenon in which one branch of a bifurcated axon has an attenuated action potential when stimulated at high frequencies. This effect has been observed with stimulation frequencies of $50 \mathrm{~Hz}{ }^{35-37}$ To minimize the potential for branch point blocking, EMG stimulation was performed at $3 \mathrm{~Hz}$. In addition to being a frequency-dependent phenomenon, branch point blocking has also occurred at sites where a single large axon bifurcates into a number of significantly smaller axons. An action potential originating at a site distal to the branch point may not propagate either into the larger parent axon due to an impedance mismatch or, subsequently, into the daughter branch. Although histomorphometric analysis of the axons in the proximal donor nerve, the distal donor nerve, and the TLN graft might potentially abrogate this problem to some degree, there appears to be no way to definitively resolve this question experimentally.

Second, our conclusions are based on a relatively small sample size. Although we did not demonstrate any electrical continuity between the right and left hindlimbs, the number of motor units in the reinnervated right hindlimb was small, raising the possibility of a type II statistical error. Future experiments will be required to confirm our findings.

It is important to note that no comparative EMG signal processing was performed, only the presence or absence of a signal was recorded. These data are qualitative and used only to evaluate the functional neuromuscular connections of axons within the left peroneal nerve and the cross leg nerve graft. Although comparative signal processing may prove useful in analyzing the efficiency of skeletal muscle reinnervation via TLN, this study was meant only to demonstrate the functional axonal anatomy underlying TLN. As a result, only electrical continuity data were necessary.

Last, it is important to note that our conclusions do not depend, in any way, on whether a "perineurial" or an "epineurial" window was created at the time of the end-to-side nerve coaptation. Previous authors have demonstrated that 
the perineurium is a barrier to axonal regeneration after TLN, ${ }^{7}$ the limited recovery of contractile function of muscles in our experiment is consistent with these data. It is likely that the efficiency of axonal regeneration and muscle reinnervation would have been improved if we had deliberately opened the perineurium of the donor left peroneal nerve. However, regardless of which investing layer of the donor peroneal nerve was windowed at the time of the TLN, we have demonstrated, unambiguously, that axons derived from the left peroneal nerve can regenerate through a TLN and establish functional neuromuscular connections in the contralateral limb. Under the conditions of our experiment, $100 \%$ of the axons that traversed the TLN graft and made synaptic contact with the right EDL and TA muscles relinquished functional connections to the same muscles in the left leg.

\section{CONCLUSIONS}

These data demonstrate that axons forming functional connections via TLN must first relinquish their original connections prior to reinnervating new motor unit territories. Therefore, as more axons regenerate through the TLN, the number of intact donor nerve axons will decrease, and partial denervation of the "donor" muscles will likely occur. The partially denervated muscles will adapt to a reduction in motor axons with an increase in the innervation ratio. ${ }^{28}$ However, if the number of motor units is reduced below a critical level, then a force deficit will likely be observed. ${ }^{38}$ Therefore, before TLN can be recommended for wide clinical application, additional studies will be required to determine the most effective technique for muscle reinnervation via TLN while maintaining an acceptable degree of donor nerve integrity.

\section{ACKNOWLEDGMENTS}

This work was supported by a grant from the Plastic Surgery Educational Foundation. Jason Rovak was supported by a grant from the University of Michigan Summer Biomedical Research program. Dr. Cederna was supported by a grant from the National Institute on Aging, Multidisciplinary Research Training in Aging, grant T32 AG00114. Dr. Macionis was supported by a grant from the J. William Fulbright Scholarship Program, Vilnius University in Lithuania. Dr. Kuzon received support as the Academic Scholar of The American Association of Plastic Surgeons. The authors thank Micrins Microsurgical Instruments, Inc. (Lake Forest, IL) for their generous contributions of microsurgical instruments for the execution of this investigation.

\section{REFERENCES}

1. Ballance CA, Ballance HA, Stewart P. Remarks on the operative treatment of chronic facial palsy of peripheral origin. Br Med J 1903;2: 1009-1013.
2. Sherren J. Some points in the surgery of the peripheral nerves. Edinb Med J 1906;20:297-332.

3. Seddon HJ. Surgical disorders of the peripheral nerves. Baltimore: Williams \& Wilkins; 1972.

4. Viterbo F, Trindade JC, Hoshino K, et al. Latero-terminal neurorrhaphy without removal of the epineural sheath: experimental study in rats. Sao Paulo Med J 1992;110:267-275.

5. Tarasidis G, Watanabe O, Mackinnon SE, Strasberg SR, Haughey BH, Hunter DA. End-to-side neurorrhaphy resulting in limited sensory axonal regeneration in a rat model. Ann Otol Rhinol Laryngol 1997;106: 506-512.

6. Noah EM, Williams A, Jorgenson C, et al. End-to-side neurorrhaphy: a histological and morphometrical study of axonal sprouting into an end-to-side nerve graft. J Reconstr Microsurg 1997;13:99-106.

7. Noah EM, Williams A, Fortes W, Terzis JK. A new animal model to investigate axonal sprouting after end-to-side neurorrhaphy. J Reconstr Microsurg 1997;13:317-325.

8. Zhao JZ, Chen ZW, Chen TY. Nerve regeneration after terminolateral neurorrhaphy: experimental study in rats. J Reconstr Microsurg 1997; 13:31-37.

9. Viterbo F, Trindade JC, Hoshino K, et al. End-to-side neurorrhaphy with removal of the epineurial sheath: an experimental study in rats. Plast Reconstr Surg 1994;94:1038-1047.

10. May M, Sobol SM, Mester SJ. Hypoglossal-facial nerve interpositional-jump graft for facial reanimation without tongue atrophy. Otolaryngol Head Neck Surg 1991;104:818-825.

11. Viterbo F, Palhares A, Franciosi LF. Restoration of sensitivity after removal of the sural nerve: a new application of lateral-terminal neurorrhaphy (case report). Rev Bras Cir Plast Est Reconst 1993;8:85-87.

12. Viterbo F. A new method for treatment of facial palsy: the cross-face nerve transplantation with end-to-side neurorrhaphy. Rev Bras Cir Plast Est Reconst 1993;8:88-91.

13. Brooks SV, Faulkner JA, McCubbrey DA. Power outputs of slow and fast skeletal muscles of mice. J Appl Physiol 1990;68:1282-1285.

14. Brooks SV, Faulkner JA. Maximum and sustained power of extensor digitorum longus muscles from young, adult and old mice. J Gerontol 1991;46:B28-31.

15. Cote C, Faulkner JA. Motor unit function in skeletal muscle autografts of rats. Exp Neurol 1984;84:292-305.

16. Faulkner JA, Niemeyer JH, Maxwell LC, White TP. Contractile properties of transplanted extensor digitorum longus muscles of cats. Am J Physiol 1980;238:C120-126.

17. Rosenblatt J, Kuzon W, Plyley M, Pynn B, McKee N. A histochemical method for the simultaneous demonstration of capillaries and fiber type in skeletal muscle. Stain Technol 1988;62:263-273.

18. Kuzon WM, Rosenblatt J, Pynn B, Plyley, Mckee N. Fiber type morphometry and capillary geometry in free, vascularized muscle transfers. Microsurgery 1991;12:352-360.

19. Brooke MH, Kaiser KK. Muscle fiber types: how many and what kind? Arch Neurol 1970;23:369-379.

20. Blomstrand E, Ekblom B. How to calculate human muscle fiber areas in biopsy samples: methodological considerations. Acta Physiol Scand 1984;122:545-551.

21. Blomstrand E, Ekblom B. The needle biopsy technique for fibre type determination in human skeletal muscle: a methodological study. Acta Physiol Scand 1982;116:437-442.

22. Sacks RD, Roy RR. Architecture of the hind limb muscles of cats: functional significance. J Morphol 1982;173:185-195.

23. Kramer H, Windrum GM. The metachromatic staining reaction. J Histochem 1955;3:227-232.

24. Foehring RC, Sypert GW, Munson JB. Properties of self-reinnervated motor units of medial gastrocnemius of cat. I. Long-term reinnervation. J Neurophysiol 1986;55:931-946.

25. Das S, Spector S, Miller T, Martin T, Edgerton V. Model for micro- 
neurovascular muscle transplantation in the dog. Plast Reconstr Surg 1986;77:804-813.

26. Stevanovic M, Seaber A, Urbaniak J. Canine experimental free muscle transplantation. Microsurgery 1986;7:105-113.

27. Karpati G, Engel W. Type grouping in skeletal muscles after experimental reinnervation. Neurology 1968;18:447-455.

28. Gordon T, Stein RB. Reorganization of motor-unit properties in reinnervated muscles of the cat. J Neurophysiol 1982;48:1175-1190.

29. Dubowitz V. Muscle biopsy: a practical approach (2nd ed.). London: WB Saunders; 1985.

30. Dettbarn WD. A distinct difference between slow and fast muscle in acetylcholinesterase recovery after reinnervation in the rat. Exp Neurol 1981;74:33-50.

31. Albani M, Lowrie MV, Vrbova G. Reorganization of motor units in reinnervated muscles of the rat. J Neurol Sci 1988;88:195-206.

32. Viterbo F, Trindade JC, Hoshino K, et al. Two end-to-side neurorrhaphies and nerve graft with removal of the epineural sheath: experimental study in rats. Br J Plast Surg 1996;47:75-80.
33. Lundborg G, Zhao Q, Kanje M, et al. Can sensory and motor collateral sprouting be induced from intact peripheral nerve by end-to-side anastomosis? J Hand Surg 1994;19B:277-282.

34. Andreopoulos GK, Skoulis TG, Terzis JK. Double labeling technique to trace axonal sprouting after end-to-side neurorrhaphy. Abstract presented at the 43rd annual meeting of the Plastic Surgery Research Council, Loma Linda, CA, April 7, 1998.

35. Parnas I. Differential block at high frequency of branches of a single axon innervating two muscles. J Neurophysiol 1972;35:903-914.

36. Stockbridge N. Theoretical response to trains of action potentials of a bifurcating axon with one short daughter branch. Biophys J 1988;54: 637-641.

37. Yau KW. Receptive fields, geometry and conduction block of sensory neurones in the central nervous system of the leech. J Physiol 1976; 23:513-538.

38. Totosy de Zepetnek JE, Van Zung H, Erdebil S, Gordon T. Innervation ratio is an important determinant of force in normal and reinnervated rat tibialis anterior muscles. J Neurophys 1995;67:1385-1403. 